\title{
A Review of Electron Channeling Contrast Imaging for Non-Destructive Defect Analysis of Crystalline Solids
}

\author{
Yoosuf N. Picard ${ }^{1}$ \\ 1. Department of Materials Science and Engineering, Carnegie Mellon University, Pittsburgh, PA, USA
}

Since its discovery in 1967 [1], electron channeling had been considered a possible route for studying crystal structures within the recently commercialized scanning electron microscope (SEM). Furthermore, it was immediately apparent that electron channeling held promise for both imaging and identifying crystallographic defects by SEM in a manner comparable to transmission electron microscopy (TEM) [2]. While unable to obtain similar resolution to TEM, SEM-based electron channeling contrast imaging (ECCI) could nevertheless access similar contrast mechanisms to TEM. Specifically, the lattice strain induced by crystallographic defects could generate contrast equivalent to TEM-based diffraction contrast. By aligning the scanned electron beam to the Bragg angle for a specific set of crystallographic planes, the detected backscattered electron (BSE) yield will strongly fluctuate near extended defects, producing BSE intensity profiles indicative of both the directionality and magnitude of local lattice displacements induced by extended defects.

However, the major limitation to growth of the ECCI technique has largely stemmed from the comparatively weak channeling intensity contrast in the acquired BSE signal as compared to effects from composition (Z-contrast) and topography. And while SEM beam rocking could produce useful electron channeling patterns [3], the electron backscattered diffraction (EBSD) method [4] supplanted electron channeling as the viable means for SEM-based crystallographic phase identification and orientation mapping. Nevertheless, ECCI application has steadily grown over the last decade due to the wide accessibility of field emission guns (FEG) SEMs as well as increasingly improved BSE detectors. Over the last 30 years, researchers have historically applied ECCI to investigate dislocation networks in relation to metal processing, forming and deformation. But more recent work has allowed robust studies of individual extended defects for low defect density specimens, like bulk crystal semiconductors and ceramics as demonstrated in Figure 1.

This presentation will review the ECCI technique, starting with the early studies of defect contrast in relation to dynamical theory of electron diffraction. This review will focus on developments in both the imaging and identification of individual linear and planar defects across a broad range of metals, semiconductors and ceramics. Examples will be presented where ECCI has facilitated new understanding on defect-property correlations as well as fundamental insights on bulk crystal growth and epitaxial thin film growth. Finally, contrast behavior for a variety of defect classes, including planar defects and linear defects, will be comprehensively summarized as a function of SEM parameters and channeling conditions.

\section{References:}

[1] DG Coates, Philosophical Magazine 16 (1967), p.1179.

[2] GR Booker, AMB Shaw, MJ Whelan and PB Hirsch, Philosophical Magazine 16 (1967), p.1185.

[3] DC Joy, DE Newbury and DL Davidson, Journal of Applied Physics 53 (1982), p.R81.

[4] DJ Dingley, Scanning Electron Microscopy 11 (1984), p.569. 

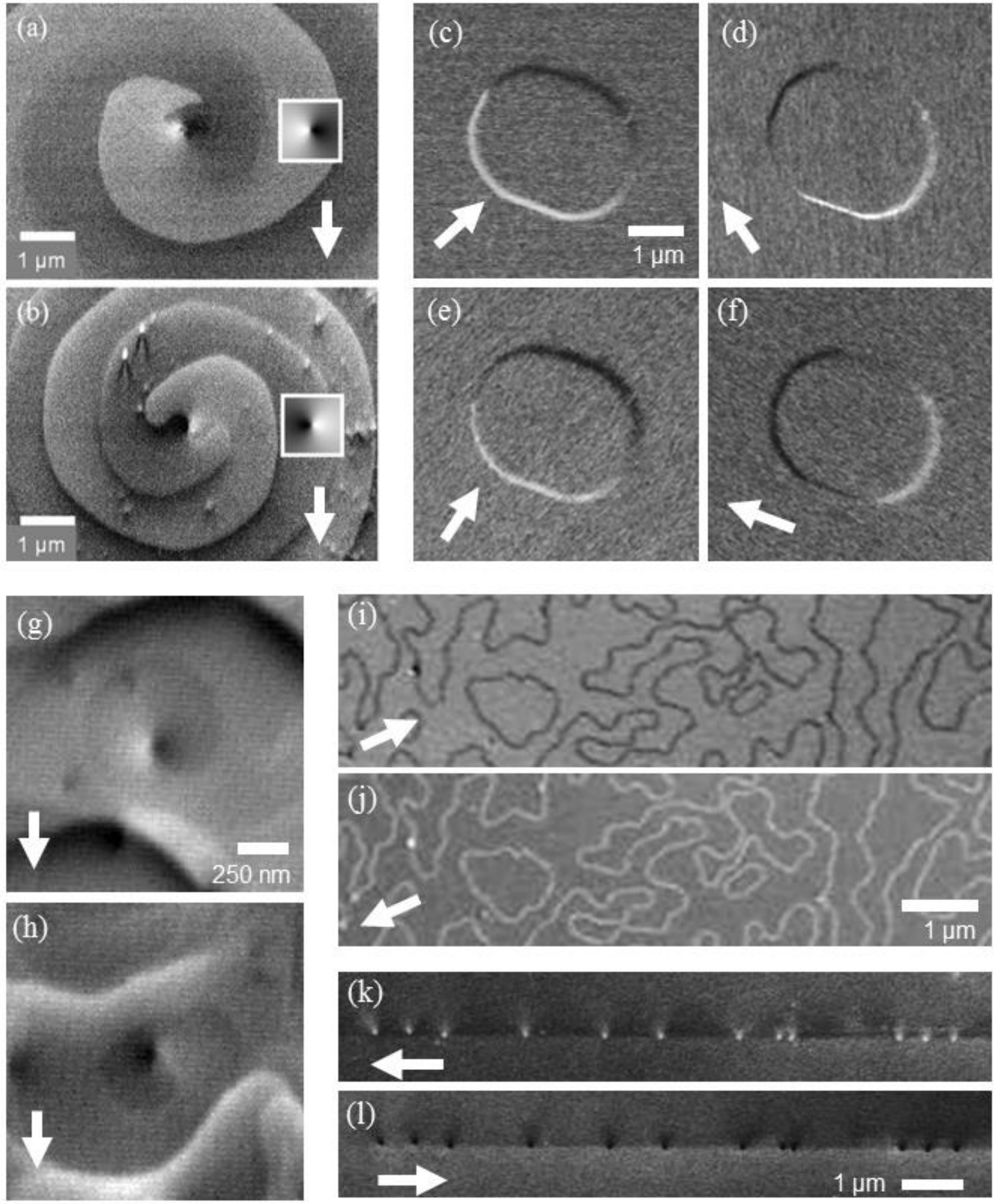

Figure 1. ECCI micrographs of $(a, b)$ opposite Burgers vector screw dislocations penetrating the surface of $4 \mathrm{H}-\mathrm{SiC}$ (0001), (c,d,e,f) a single sub-surface dislocation loop in $\mathrm{SrTiO}_{3}(001)$, (g,f) opposite Burgers vector screw dislocations penetrating the surface of $\mathrm{GaN}(0002),(\mathrm{i}, \mathrm{j})$ anti-phase domain boundaries in a $\mathrm{La}_{0.7} \mathrm{Sr}_{0.3} \mathrm{MnO}_{3}$ (110) surface, $(\mathrm{k}, \mathrm{l})$ an array of dislocations in a $\mathrm{Ga}_{2} \mathrm{O}_{3}(-201)$ bulk crystal surface. White arrows in each micrograph denotes the direction of the acting diffraction vector. 